\title{
Particle Focusing in Microchannel with Multi-Parallel Channels or Porous Orifice
}

\author{
Hiroshige Kumamaru ${ }^{1, ~ *, ~ H i r o f u m i ~ S u g a m i ~}{ }^{1}$, Masaki Nakahira ${ }^{2}$, Naohisa Takagaki ${ }^{1}$ \\ ${ }^{1}$ Department of Mechanical Engineering, University of Hyogo, Himeji, Japan \\ ${ }^{2}$ Department of Mechanical and System Engineering, University of Hyogo, Himeji, Japan
}

Email address:

sphb3u59@true.ocn.ne.jp(H. Kumamaru)

${ }^{*}$ Corresponding author

\section{To cite this article:}

Hiroshige Kumamaru, Hirofumi Sugami, Masaki Nakahira, Naohisa Takagaki. Particle Focusing in Microchannel with Multi-Parallel Channels or Porous Orifice. International Journal of Mechanical Engineering and Applications. Vol. 6, No. 3, 2018 , pp. 46-54. doi: $10.11648 /$ j.ijmea.20180603.11

Received: April 22, 2018; Accepted: May 7, 2018; Published: May 28, 2018

\begin{abstract}
The sheathless particle focusing in a microchannel installing one set of multi-parallel channels or one porous orifice is proposed in this study. Numerical calculations have been carried out by the Lagrange two-phase model (i.e. the particle tracking model) in PHOENICS software for the microchannel including the porous orifice, in simulating both the channels installing the multi-parallel channels and the porous orifice. It becomes clear from calculation results that the particle focusing may be achieved at lower inlet velocities by using the porous orifice. Low velocities correspond to reduction in the sample flow including particles (i.e. cells). It also becomes obvious that the particle focusing is improved if the porous orifice with taper shape is used. Furthermore, particle focusing experiments have been conducted in the channel including the multiparallel channels. It becomes clear that the particle focusing may be achieved by using the multi-parallel channels.
\end{abstract}

Keywords: Particle Focusing, Microchannel, Multi-Parallel Channels, Porous Orifice

\section{Introduction}

Particle focusing into a narrow stream in a microchannel is an important step in flow cytometry system (i.e. flow cytometer) to be used in both biomedical research and clinical diagnostics [1-3]. In the flow cytometer, analyses are performed by passing a narrow particle stream under a focused laser beam in order to obtain information on the size, type and content of cells at rates more than hundreds of particles (i.e. cells) per seconds.

The particle focusing methods are classified into sheath flow focusing and sheathless focusing. Figure 1 explains the sheath flow focusing, in which two horizontal sheath flows force particles in the sample flow to flow in a narrow stream [4]. However, in order to decrease the flow rates of sheath flows and simplify the focusing device (i.e. flow cell), various sheath flow focusing methods which combine the sheath flows with various hydrodynamic forces induced by channel geometry or configuration have been studied. In these methods, the sheath flow focusing is promoted by hydrodynamic forces induced by a curved channel [5], a curved channel together with sharp corner structures [6], orifices [7], a channel with chevrons (i.e. V-shaped blocks) $[8,9]$, or a twisted channel [10].

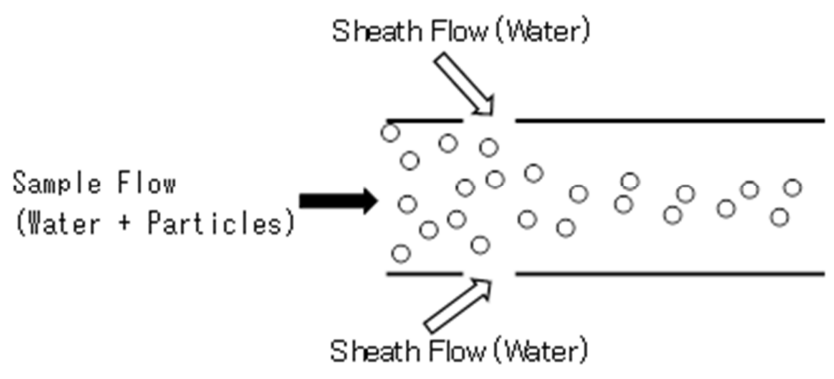

Figure 1. Particle focusing by sheath flow.

In order to further decrease the flow rates of sheath flows and simplify the focusing device, various sheathless focusing methods have been proposed. The sheathless focusing relies on externally applied forces or internally induced forces by channel geometry or configuration. The former focusing is 
applicable to limited particles which are affected by electric field and others. As the latter focusing, methods using multiple curved channels or serpentine channels [11-20], a channel with triangular cross-section [21], a channel equipped with orifices [22], or a channel installed with V-shaped blocks [23] have been proposed.

In this study, sheathless focusing in a microchannel by using one set of multi-parallel channels or one porous orifice is proposed, and studied by numerical calculations and experiments. The present focusing method may be applicable to low flow rates of the sample flow including particles. The low flow rates correspond to saving of examining particles (i.e. cells).

\section{Numerical Calculations}

\subsection{Proposed Particle Focusing}

The sheathless focusing methods by using one set of multiparallel channels and one porous orifice in microchannels, shown in Figures 2(a) and 2(b), respectively, are proposed in this study. In the multi-parallel channels, particles can pass through the central cannel with a large width, though particles cannot flow in the other channels with a small width. As a result, the particles may flow in a narrow stream in the downstream of the multi-parallel channels. In the porous orifice, since the orifice is made of porous material, fluid can pass through the orifice walls and the orifice opening, though particles can flow only through the orifice opening. As a result, the particles also may focus in the center region downstream of the porous orifice.

\subsection{Calculation Models}

Commercial thermal fluid analysis software usually includes an Euler two-phase model (i.e. two-fluid model) and a Lagrange two-phase model (i.e. particle tracking model). Both two-phase models equipped in commercial software usually cannot treat the situation in which particles cannot pass through a channel having a width smaller than particle diameters or porous orifice walls. However, the Lagrange two-phase model (i.e. particle tracking model) in PHOENICS software [24] can deal with the impossibility of passage through porous material by setting the threshold of particle passage for the porous material.

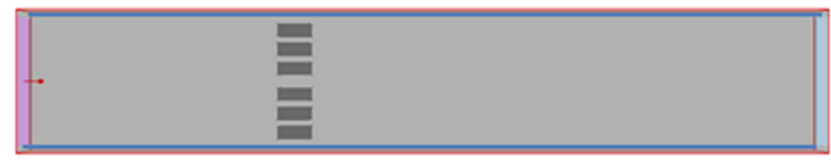

(a) Multi-parallel channels.

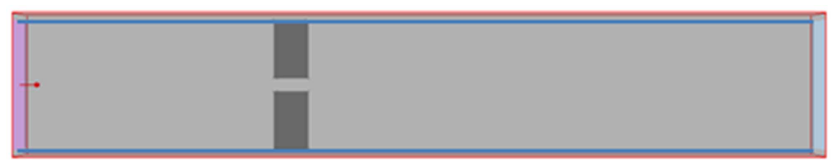

(b) Porous orifice.

Figure 2. Proposed microchannels.
For this reason, in the present research, the Lagrange model in PHOENICS has been used to study the particle focusing in a microchannel with a porous orifice. In the Lagrange model, the equations of mass conservation and momentum conservation for liquid phase, and the equation of motion for particles are solved together with the constitutive equation for the interphase drag between liquid phase and particles.

Numerical calculations have been performed for the microchannel with one porous orifice shown in Figure 2(b). However, considering execution of experiments explained later, base case calculations have been carried out for a channel ten times larger than an original channel. The base case model and the original channel are called "base case channel" and "original microchannel," respectively, in this paper. Table 1 compares dimensions and inlet velocity of these two channels. The base case channel is enlarged from the original microchannel, in keeping the Reynolds number $(R e=u d / v)$ and the particle Reynold number $\left(R e_{\mathrm{p}}=u d_{\mathrm{p}} / v\right)$. Here, $u$ is the flow velocity, $v$ the kinematic viscosity of fluid, $d$ the equivalent diameter of channel, $d_{\mathrm{p}}$ the diameter of particle. The calculations are carried out by using 35, 20 and 100 grid elements in the width, height and length directions, respectively, closely spaced near the orifice.

Table 1. Dimensions and inlet velocity in base case channel and original microchannel.

\begin{tabular}{lll}
\hline & Base Case Channel & Original Microchannel \\
\hline $\begin{array}{l}\text { Channel } \\
\text { Length }(\mu \mathrm{m})\end{array}$ & 12500 & 1250 \\
Width $(\mu \mathrm{m})$ & 2000 & 200 \\
Height $(\mu \mathrm{m})$ & 500 & 50 \\
Orifice & \\
Opening $(\mu \mathrm{m})$ & 200 & 20 \\
$\begin{array}{l}\text { Thickness } *(\mu \mathrm{m}) \\
\text { Porosity }\end{array}$ & 500 & 50 \\
$\begin{array}{l}\text { Particle } \\
\text { Diameter }(\mu \mathrm{m})\end{array}$ & 0.5 & 0.5 \\
$\begin{array}{l}\text { Inlet Condition } \\
\text { Velocity }(\mathrm{cm} / \mathrm{s})\end{array}$ & 100 & 10 \\
\hline
\end{tabular}

* Thickness of orifice plate (wall)

\subsection{Calculation Results in Base Case}

Base case calculations have been performed for the base case channel. Water including polystyrene particles (with diameters of $100 \mu \mathrm{m}$ and volume fraction of $2 \%$ ) enters the channel at an inlet velocity of $5 \mathrm{~cm} / \mathrm{s}$. Figures 3(a), 3(b) and 3(c) show calculation results of the pressure, the velocity (magnitude) and the particle trajectory, respectively for the base case channel.

As shown in Figure 3(a), the pressure decreases along the flow channel. The pressure does not drop steeply through the orifice, since the orifice is made of porous material with porosity of 0.5 . The water passes through not only the orifice opening but also the porous orifice walls, as seen in Figure 3(b). The water velocity increases within and just downstream of the orifice opening and the orifice walls, since the flow cross section decreases in these regions. As shown 
in Figure 3(c), the particles pass only through the orifice opening, and the particles flow in a narrow region downstream of the orifice while they spreads to some degree along the channel. The particles are injected from $10 \times 2$ points into the channel at the same velocity as the water. The color scale in the figure represents the particle travelling time from the channel inlet to each point.

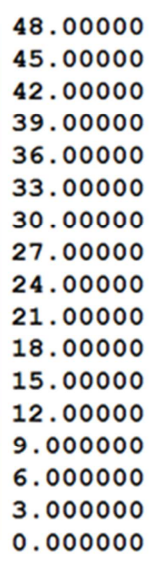

0.224000

0.210000

0.196000

0.182000

0.168000

0.154000

0.140000

0.126000

0.112000

0.098000

0.084000

0.070000

0.056000

0.042000

0.028000

0.014000

0.000000

0.586876

0.550197

0.513517

0.476837

0.440157

0.403477

0.366798

0.330118

0.293438

0.256758

0.220079

0.183399

0.146719

0.110039

0.073360

0.036680

0.000000

Pressure, Pa

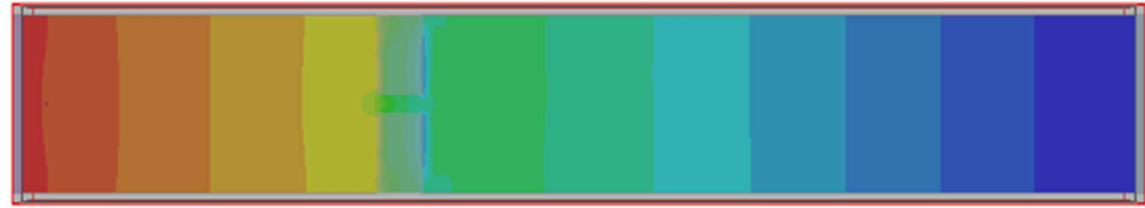

(a) Pressure

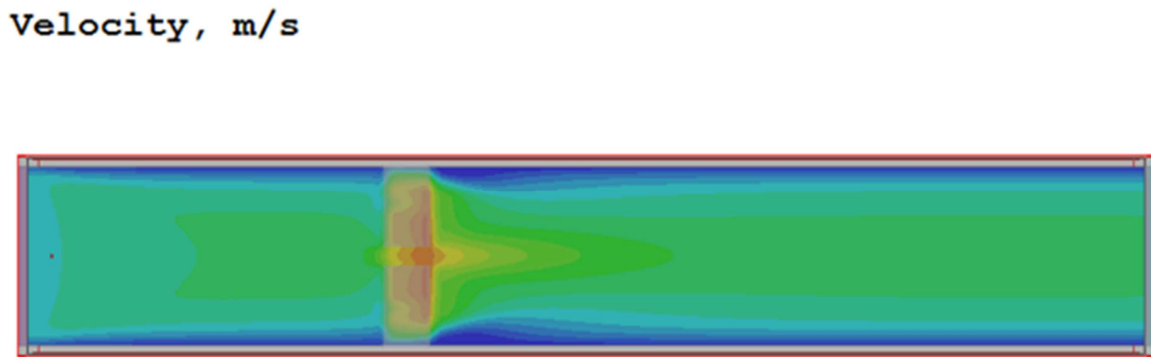

(b) Velocity

Total time (s)

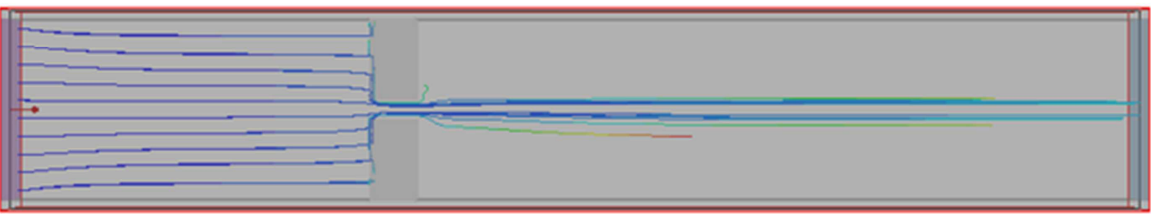

(c) Particle trajectory

Figure 3. Calculation results in base case.

\subsection{Calculation Results in Low Velocity Cases}

Numerical calculations have been carried out for lower inlet velocities. Figures 4(a), 4(b) and 4(c) show calculated particle trajectories in the cases of inlet velocities of $2.5 \mathrm{~cm} / \mathrm{s}, 1.0 \mathrm{~cm} / \mathrm{s}$ and $0.5 \mathrm{~cm} / \mathrm{s}$, respectively. As shown in Figure 3(c) (the base case, $5 \mathrm{~cm} / \mathrm{s}$ ) and Figures 4(a) through 4(c), the particle focusing downstream of the orifice is achieved at low velocities down to $0.5 \mathrm{~cm} / \mathrm{s}, 1 / 10$ of base case, while the particles spread gradually in lower velocities. Low velocities correspond to reduction in 
the sample flow including particles (i.e. cells).

\subsection{Calculation Results in Small Scale Cases}

Next, numerical calculations have been performed for smaller scale channels. Figure 5(a) shows calculated particle trajectories in the channel five times larger than the original microchannel, while Figure 5(b) gives those in the original microchannel itself. Comparing the trajectories in Figure 3(c) (the base case channel, 10 times larger than the original microchannel), Figure 5(a) (the channel 5 times larger than the original microchannel) and Figure 5(b) (the original microchannel), it is obvious the particle focusing downstream of the orifice becomes unclear in a smaller scale channel, i.e. particularly in the original microchannel.
0.492010
0.461259
0.430508
0.399758
0.369007
0.338257
0.307506
0.276755
0.246005
0.215254
0.184504
0.153753
0.123002
0.092252
0.061501
0.030751
0.000000

0.961979

0.901855

0.841732

0.781608

0.721484

0.661361

0.601237

0.541113

0.480990

0.420866

0.360742

0.300618

0.240495

0.180371

0.120247

0.060124

0.000000

1.904256

1.785240

1.666224

1.547208

1.428192

1.309176

1.190160

1.071144

0.952128

0.833112

0.714096

0.595080

0.476064

0.357048

0.238032

0.119016

0.000000

\section{Total time (s)}

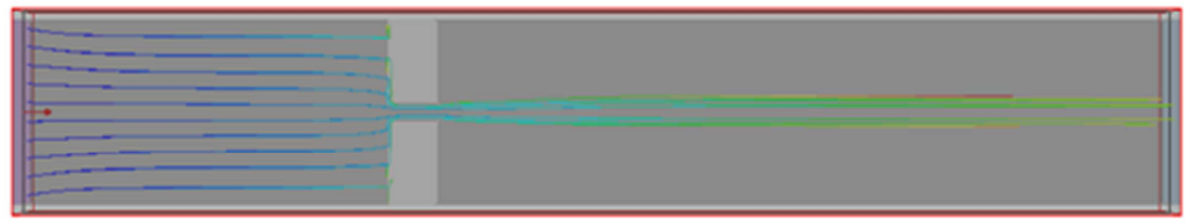

(a) $\mathrm{u}=2.5 \mathrm{~cm} / \mathrm{s}$

Total time (s)

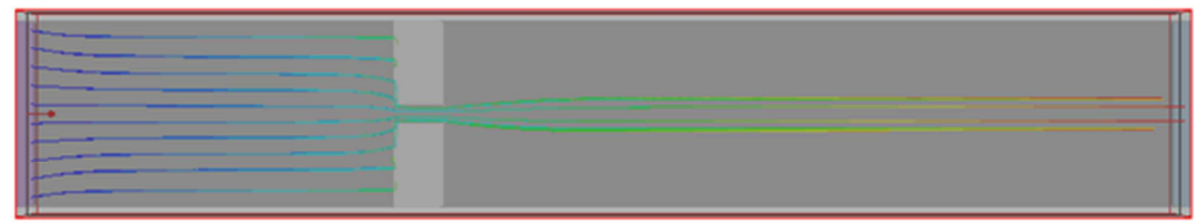

(b) $\mathrm{u}=1.0 \mathrm{~cm} / \mathrm{s}$

Total time (s)

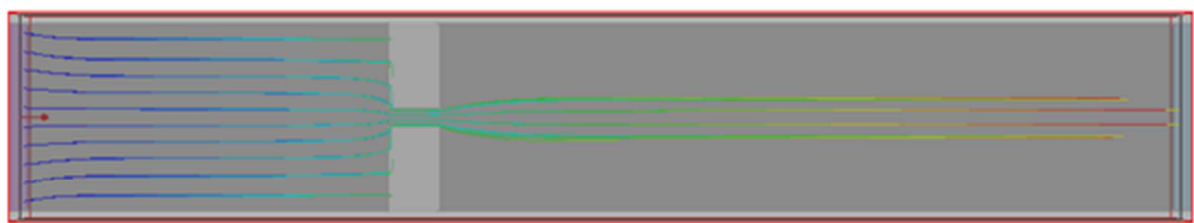

(c) $\mathrm{u}=0.5 \mathrm{~cm} / \mathrm{s}$

Figure 4. Calculated trajectories in low velocity cases. 
0.065970

0.061847

0.057724

0.053600

0.049477

0.045354

0.041231

0.037108

0.032985

0.028862

0.024739

0.020616

0.016492

0.012369

0.008246

0.004123

0.000000

Total time (s)

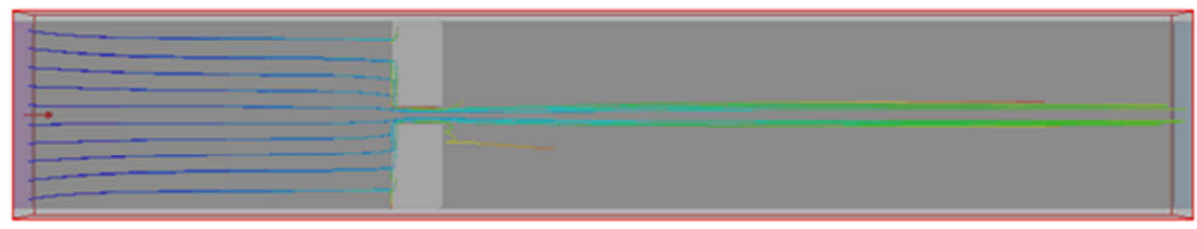

(d) In channel 5 times larger than original microchannel
0.027928
0.026183
0.024437
0.022692
0.020946
0.019201
0.017455
0.015710
0.013964
0.012219
0.010473
0.008728
0.006982
0.005237
0.003491
0.001746
0.000000

Total time (s)

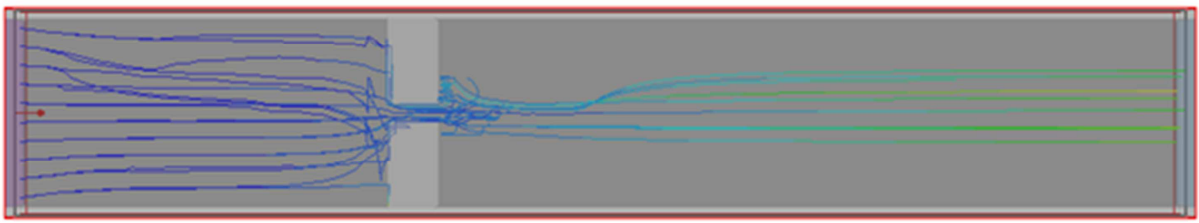

(e) In original microchannel

Figure 5. Calculated trajectories in small scale cases.

0.024869

0.023315

0.021760

0.020206

0.018652

0.017097

0.015543

0.013989

0.012434

0.010880

0.009326

0.007772

0.006217

0.004663

0.003109

0.001554

0.000000

\section{Total time (s)}

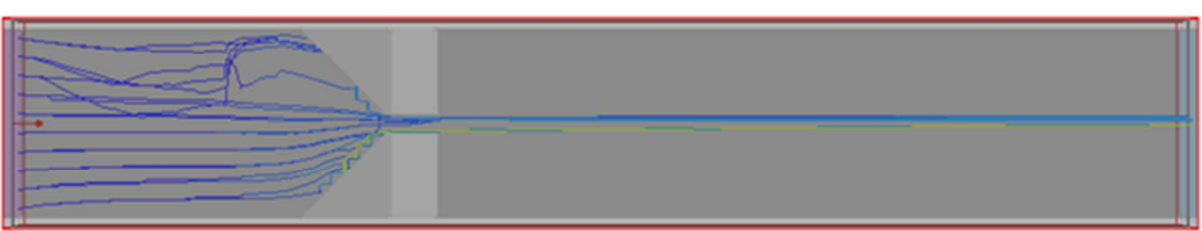

Figure 6. Calculated trajectories in original microchannel with taper-shape orifice.

In order to improve the particle focusing in the original microchannel, numerical calculations have been conducted for an original microchannel in which the porous orifice has a taper (45 degree) shape in the upstream side of the orifice, as shown in Figure 6. Figure 6 gives the particle trajectory in the original microchannel with the tapered orifice. Comparing the results in Figure 5(b) (without the taper shape) and Figure 6 (with the taper shape), it becomes clear that the particle focusing is improved fairly by installing the taper shape.

From the calculation results explained above, it is considered that the particle focusing can be achieved in the original microchannel with one porous orifice. Also, this particle focusing may be kept at lower liquid velocities. Low velocities correspond to reduction in the sample flow including particles (i.e. cells). 


\section{Experiments}

\subsection{Experimental Channel and Experimental Method}

Particle focusing experiments have been performed by using a flow channel shown in Figure 7, called "experimental channel" in this paper. The experimental channel, with a length of $38 \mathrm{~mm}$, a width of $4 \mathrm{~mm}$ and a height of $1 \mathrm{~mm}$, is equipped with one set of multi-parallel channels having one large channel with a width of $0.4 \mathrm{~mm}$ and six small channels with a width of $0.1 \mathrm{~mm}$. The flow channels and inlet/outlet were fabricated on upper and lower acryl plates, respectively, by machinery cutting with an end-mill. The upper and lower acryl plates are attached by burning in an electric furnace at $110 \mathrm{C}$ during 5 hours. Water including fluorescent polystyrene particles, with diameters of $200 \mu \mathrm{m}$ and volume fraction of $0.2 \%$, is used as a sample fluid.

Figure 8 shows the experimental setup. By a syringe pump, the water including the particles enters the experimental channel, at a constant velocity of $2.5 \mathrm{~cm} / \mathrm{s}$, and leaves the channel after passing through the parallel channels. The particle trajectories including the particle focusing behavior are observed by a video camera (1920 x 1080 pixel, $60 \mathrm{fps}$ ) under lighting by LED light source.
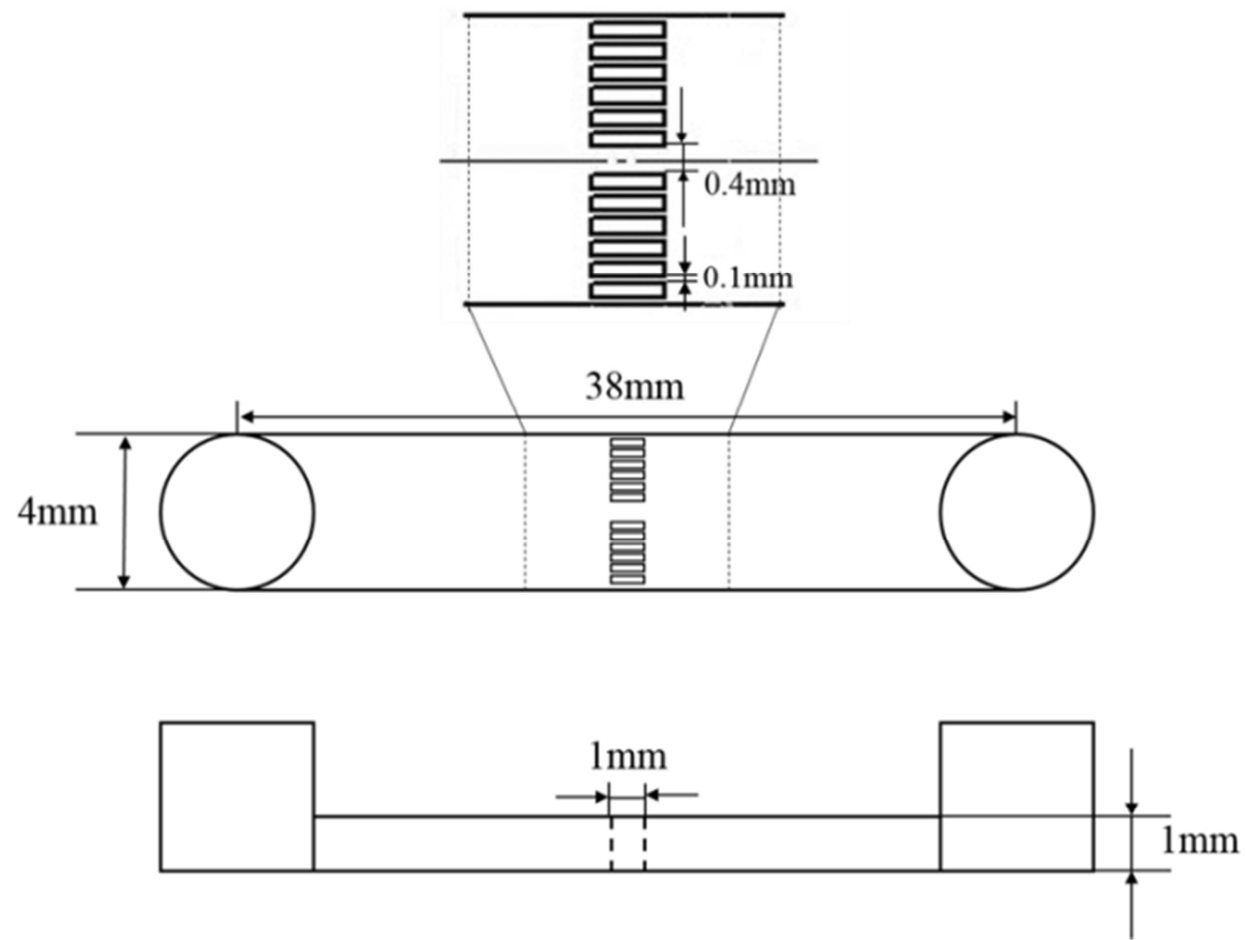

Figure 7. Experimental channel.

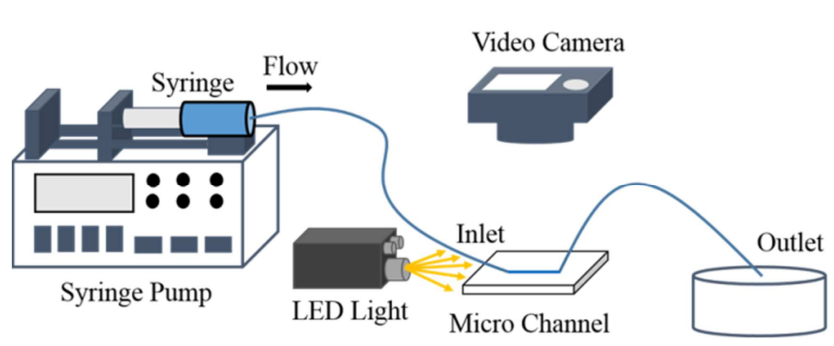

Figure 8. Experimental setup.

\subsection{Numerical Calculations for Experimental Channel}

Numerical calculations have been performed for the experimental channel, since the experimental channel is twice as large as the base case channel. Table 2 compares the experimental channel and the base case channel. Note that the inlet velocity is halved to $2.5 \mathrm{~cm} / \mathrm{s}$ in order to keep the Reynold number. Corresponding to experimental geometry and conditions, the porosity of the orifice walls is changed to 0.33 , and the volume fraction of particles is set to $0.2 \%$.

Figures 9(a) and 9(b) give calculated particle trajectories for the experimental channels without taper shape and with taper shape (45 degree), respectively. Figures 9(a) and 9(b) show the particle focusing in the downstream of the orifice is achieved in the experimental channel without and with taper shape. The passing time from the inlet to the outlet in the channel is roughly halved by installing the taper shape. (Almost no difference was found in calculation results between particle volume fractions of $0.2 \%$ and $2 \%$ ) 
Table 2. Dimensions and inlet velocity in experimental channel.

\begin{tabular}{lll}
\hline & Experimental Channel & Base Case Channel \\
\hline Channel & & \\
Length $(\mu \mathrm{m})$ & $(38000)$ & 12500 \\
Width $(\mu \mathrm{m})$ & 4000 & 2000 \\
Height $(\mu \mathrm{m})$ & 1000 & 500 \\
Orifice & & 200 \\
Opening $(\mu \mathrm{m})$ & 400 & 500 \\
Thickness* $(\mu \mathrm{m})$ & 1000 & 0.5 \\
Porosity & 0.33 & 100 \\
Particle & & \\
Diameter $(\mu \mathrm{m})$ & 200 & 5 \\
Inlet Condition & & \\
Velocity $(\mathrm{cm} / \mathrm{s})$ & 2.5 & \\
\hline
\end{tabular}

* Thickness of orifice plate (wall)

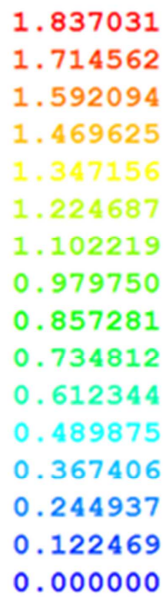

0.988690

0.922778

0.856865

0.790952

0.659127

0.593214

0.527302

0.461389

0.395476

0.329563

0.263651

0.197738

0.131825

0.065913

0.000000

\section{Total time (s)}

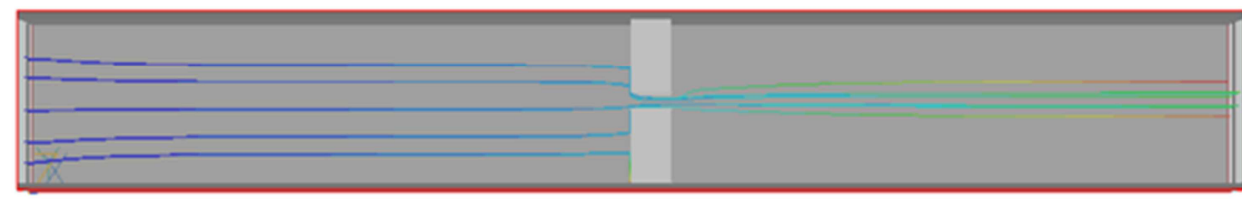

(a) For channel without taper shape

\section{Total time (s)}

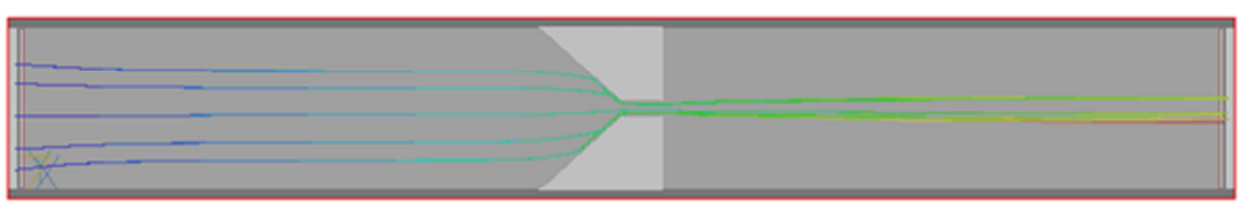

(b) For channel with taper shape

Figure 9. Calculated trajectories in experimental channel.

\subsection{Experimental Results}

Figure 10 presents particle distribution images obtained in the experimental channel (installing the orifice, i.e. the parallel channels, without taper shape) by the video camera at each $0.1 \mathrm{~s}$ for $0.8 \mathrm{~s}$. As shown in Figure 10, while the particles spread in the whole channel in the upstream of the orifice, i.e. the parallel channels, the particles flow in the narrow stream in the downstream of the orifice.
However, the particles also remain just upstream of the orifice. For this reason, it was tried to use the experimental channel installing the parallel channels with taper shape; however, the parallel channels with taper shape could not been fabricated by machinery cutting with an end-mill. Thus, it is desirable to produce the parallel channels by other methods such as MEMS manufacturing method. 


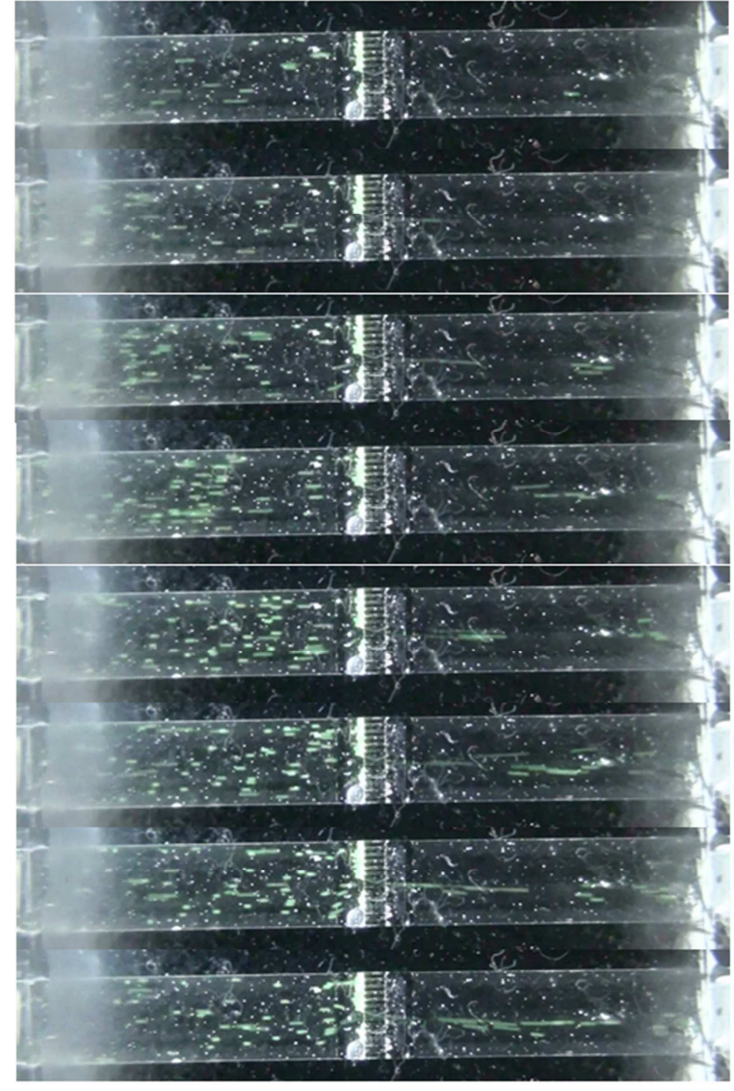

Figure 10. Particle distribution images.

\section{Conclusion}

The sheathless particle focusing in a microchannel by using one set of multi-parallel channels or one porous orifice is studied by numerical calculations and experiments.

First, numerical calculations have been carried out for the latter focusing method in simulating both the focusing methods. It becomes clear that the particle focusing is achieved in the "base case channel," ten times larger than the "original microchannel." Secondly, numerical calculations have been performed at lower inlet velocities down to $1 / 10$ of the original case. The particle focusing is kept in these low inlet velocities. Low velocities correspond to reduction in the sample flow including particles (i.e. cells). Thirdly, numerical calculations have been carried out in smaller scales down to the original microchannel. It becomes clear that the particle focusing is achieved even in the original microchannel, if the porous orifice with taper shape is used.

Particle focusing experiments have also been conducted in the channel having nearly the same size as the base case channel including the multi-parallel channels. It was proved that the particle focusing may be achieved by the multiparallel channels.

\section{References}

[1] D. D. Carlo, "Critical review: Inertial microfluidics," Lab on a Chip, vol. 9, pp. 3038-3046, 2009.
[2] X. Xuan, J. Zhu, and C. Church, "Review: Particle focusing in microfluidic devices," Microfluid Nanofluid, vol. 9, pp. 1-16, 2010.

[3] J. M. Martel, and M. Toner, "Inertial Focusing in Microfluidics,” Annu Rev Biomed Eng., vol. 16, pp. 371-396, 2014.

[4] N. Watkins, B. M. Venkatesan, M. Toner, W. Rodriguez, and R. Bashir, "A robust electrical microcytometer with 3dimensional hydrofocusing," Lab on a Chip, vol. 9, pp. 3177 3184,2009

[5] X. Mao, S. C. S. Lin, C. Dong, and T. J. Huang, "Single-layer planar on-chip flow cytometer using microfluidic drifting based three-dimensional (3D) hydrodynamic focusing," Lab on a Chip, vol. 9, pp. 1583-1589, 2009.

[6] L. L. Fan, X. K. He, Y. Han, J. Zhe, and L. Zhao, "Continuous 3D particle focusing in a microchannel with curved and symmetrical sharp corner structures," J. of Micromechanics and Microengineering, vol. 25, no. 3, 035021, 2015.

[7] M. G. Lee, S. Choi, and J. K. Park, "Three-dimensional hydrodynamic focusing with a single sheath flow in a singlelayer microfluidic device," Lab on a Chip, vol. 9, pp. 3155$3160,2009$.

[8] J. P. Golden, J. S. Kim, J. S. Erickson, L. R. Hilliard, P. B. Howell, G. P. Anderson et al., "Multi-wavelength microflow cytometer using groove-generated sheath flow," Lab on a Chip, vol. 9, pp. 1942-1950, 2009.

[9] N. Hashemi, P. B. Howell, J. S. Erickson, J. P. Golden, and F. S. Ligler, "Dynamic reversibility of hydrodynamic focusing for recycling sheath fluid," Lab on a Chip, vol. 10, pp. 19521959,2010

[10] T. Sato, and R. Miyake, "Sheath flow forming by using twisted micro-channel," Transactions of The Japan Society of Mechanical Engineering (JSME), vol. 80, no. 813, pp. 1-11, 2014 [In Japanese].

[11] D. D. Carlo, J. F. Edd, D. Irimia, R. G. Tompkins, and M. Toner, "Equilibrium separation and filtration of particles using differential inertial focusing," Analytical Chemistry, vol. 80, no. 6, pp. 2204-2211, 2008.

[12] D. R. Gossett, and D. D. Carlo, "Particle focusing mechanisms in curving confined flows," Analytical Chemistry, vol. 81, no. 20, pp. 8459-8465, 2009.

[13] J. Oakey, R. W. Applegate, E. Arellano, D. D. Carlo, S. W. Graves and M. Toner, "Particle focusing in staged inertial microfluidic devices for flow cytometry," Analytical Chemistry, vol. 82, no. 9, pp. 3862-3867, 2010.

[14] J. M. Martel, and M. Toner, "Particle focusing in curved microfluidic channels," Scientific Reports 3, 3340 (8 pages), 2013.

[15] J. Zhang, W. Li, M. Li, G. Alici, and N. Nguyen, "Particle inertial focusing and its mechanism in a serpentine microchannel," Microfluidics and Nanofluidics, vol. 17, no. 2, pp. 305-316, 2014.

[16] A. Ozbey, M. Karimzadehkhouei, S. Akgonul, D. Gozuacik, and A. Kosar, "Inertial focusing of microparticles in curvilinear microchannels," Scientific Reports 6, 38809 (11 pages), 2016. 
[17] D. Jiang, W. Tang, N. Xiang, and Z. Ni, "Numerical simulation of particle focusing in a symmetrical serpentine microchannel," RSC Advances, vol. 6, issue 62, pp. 5764757657, 2016.

[18] P. Paie, F. Bragheri, D. D. Carlo, and R. Osellame, "Particle focusing by 3D inertial microfluidics," Microsystem \& Nanoengineering, vol. 3, 17027 (8 pages), 2017.

[19] L. Wang, and D. S. Dandy, "High-throughput inertial focusing of micrometer- and sub-micrometer-sized particles separation," Advanced Science, vol. 4, 1700153 (11 pages), 2017.

[20] A. Shamloo, and A. Mashhadian, "Inertial particle focusing in serpentine channels on a centrifugal platform," Physics of Fluids, vol. 30, no. 1, 012002, 2018.
[21] J. A. Kim, J. R. Lee, T. J. Je, E. C. Jeon, and W. Lee, "Sizedependent inertial focusing position shift and particle separations in triangular microchannels," Analytical Chemistry, vol. 90, no. 3, pp. 1827-1835, 2018.

[22] J. S. Park, S. H. Song, and H. I. Jung, "Continuous focusing of microparticles using inertial lift force and velocity via multiorifice microfluidic channels," Lab on a Chip, vol. 9, pp. 939948, 2009.

[23] S. Choi, and J. K. Park, "Sheathless hydrophoretic particle focusing in a microchannel with exponentially increasing obstacle arrays," Analytical Chemistry, vol. 80, no. 8, pp. 3035-3039, 2008.

[24] http://www.cham.co.uk/ 